\title{
Tips de eco-Doppler para la evaluación de la aorta abdominal
}

\author{
Gabriel Perea* \\ Adrián H. D’Ovidio ** \\ Patricio Glenny ** \\ Corneli Mariana
}

* Instituto Cardiovascular de Buenos Aires. Argentina

** Hospital Rauwson, San Juan. Argentina

*** Instituto Cardiovascular Adventista. Argentina

****Instituto de Cardiología de la Plata. ICBA. Argentina

Recibido: $x x / x x / 2020$

Aceptado: $x x / x x / 2020$

En línea: 31/12/2020
Correspondencia

Gabriel Perea

pereagabriel@gmail.com

Citar como: Perea G. Tips de Eco-Doppler para la evaluación de la aorta abdominal (RETIC). 2020 (Dic); 3 (3): 60-61. doi: 10.37615/retic.v3n3a17.

Cite this as: Perea G. Eco-Doppler Tips for the evaluation of the abdominal aorta (RETIC). 2020 (Dic); 3 (3): 60-61. doi: 10.37615/retic.v3n3a17.

\section{Palabras clave \\ $\triangleright$ Aorta}

$\triangleright$ Doppler vascular

\section{Keywords}

$\triangleright$ Aorta

$\triangleright$ Vascular Doppler

\section{RESUMEN}

La Ecografía Doppler nos permite estudiar la Aorta Abdominal y de esta manera se puede determinar la presencia de lesiones como aneurismas, pseudoaneurismas, estenosis, trombos, placas y vasos ectásicos

\section{ABSTRACT}

Doppler ultrasound allows us to study the Abdominal Aorta so that the presence of lesions such as aneurysms, pseudoaneurysms, stenosis, thrombi, plaques and ectatic vessels can be determined.

\section{¿Cómo se realiza la exploración? Tips and tricks}

- Aunque se recomienda ayuno de 6 horas, en la mayoría de los casos se puede tener una visualización satisfactoria para medir diámetros aún sin preparación, lo cual lo hace practicable en pacientes que concurren para realización de un ecocardiograma y están en edad de screening de aneurisma (cribado oportunista).

- Se debe seleccionar el transductor apropiado al hábito constitucional del paciente. Lo habitual es el convexo (3-5 MHz) o el sectorial (3-4 MHz) que se utiliza en ecocardiografía.

- El paciente se colocará en decúbito dorsal, comenzando el estudio desde la zona subxifoidea, identificando las diferentes estructuras con el transductor en sentido longitudinal, basculando el mismo sobre la línea media del abdomen ${ }^{(1)}$, recorriendo el vaso en sentido caudal hasta llegar a su bifurcación ilíaca.

- La aorta se sitúa anteriormente a la columna vertebral y algo a la izquierda de la línea media en su recorrido intra-abdominal. Por lo tanto, la Aorta y la vena cava inferior se diferencian fácilmente ${ }^{(2)}$.

- La exploración longitudinal nos permitirá identificar la Arteria Mesentérica Superior (AMS) habitualmente a la altura de la Arteria Renal Derecha (ARD) dividiendo la aorta en yuxta-renal (en el mismo plano de la AMS o ARD), infra-renal y supra-renal proximal a la misma.
Tips para no olvidar

- Uno de los puntos clave del eco Doppler de aorta abdominal es la realización de mediciones del diámetro del vaso.

- Realizar las mediciones en cortes transversos (Figuras 1 y 2).

- Se deben realizar medidas del diámetro de la aorta a nivel supra, yuxta e infrarrenal, y examinar hasta su bifurcación en las arterias iliacas (Figura 3),

- Dichas medidas se realizan en sentido antero-posterior, de borde externo a borde externo del vaso (de adventicia a adventicia) (Figuras 4 y 5 ).

- El diámetro máximo de la aorta es de $20 \mathrm{~mm}$. en los adultos y varia poco con la edad, el sexo, la raza y el hábito corporal(3).

- Las arterias iliacas normales tienen bordes lisos y calibre uniforme. El diámetro máximo de la arteria iliaca común (de pared externa a pared externa) es de $10 \mathrm{~mm}$, y la arteria iliaca externa es algo más pequeña (4, 5) (Figura 3).

- Con el Doppler color y pulsado se determinarán las características del flujo en cada uno de los sitios antes citados.

- El volumen de muestra utilizado para el Doppler pulsado debe ser de 1,5 a $2 \mathrm{~mm}$.

Se coloca en el centro del vaso, usando un ángulo apropiado de $60^{\circ}$ (6) lo que permite obtener un patrón espectral trifasico en condiciones de normalidad. 


\section{- Trukipedia Truco 04}

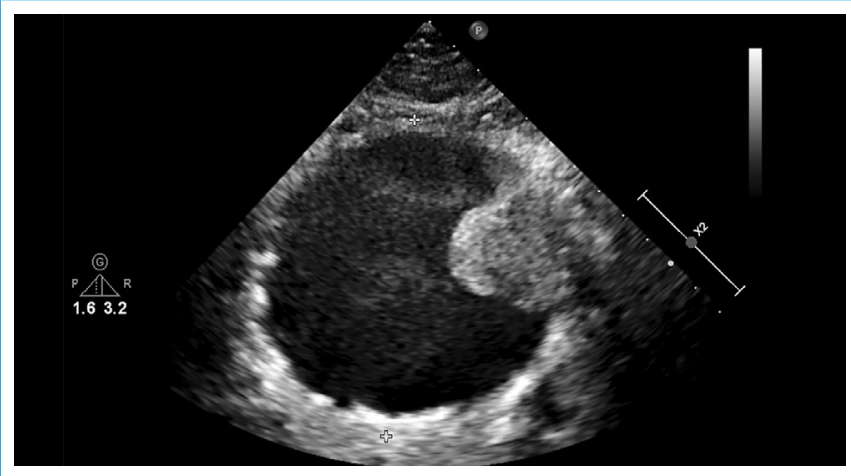

Figura 1. Corte transverso de Aneurisma de Aorta Abdominal con trombo

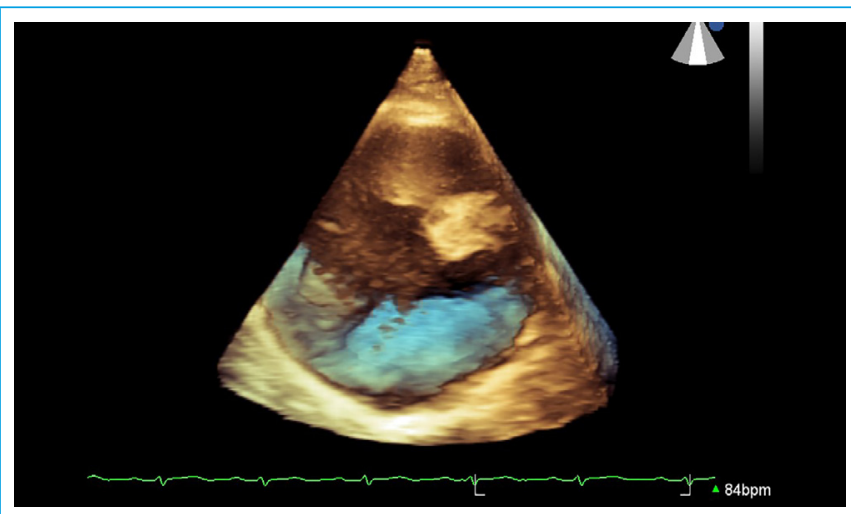

Figura 2. Imagen tridimensional de Aneurisma de Aorta Abdominal con trombos fijos y ectasia

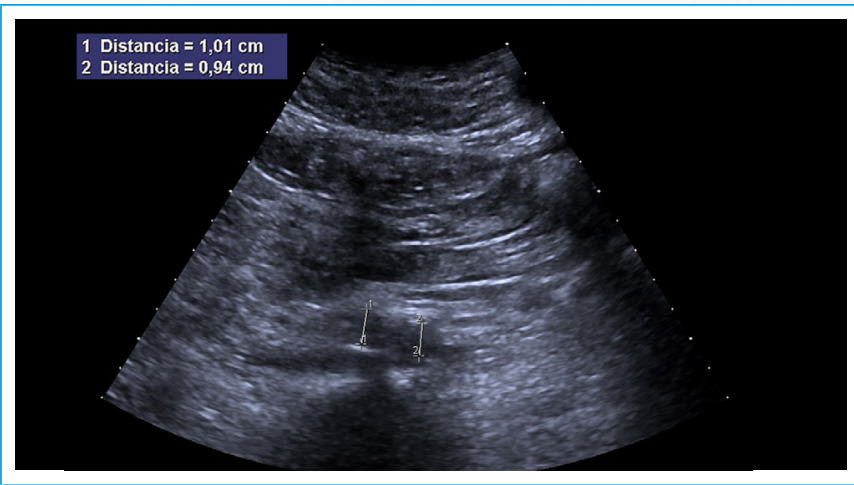

Figura 3. Corte transverso de arterias iliacas primitivas: medición de diámetro antero-posterior

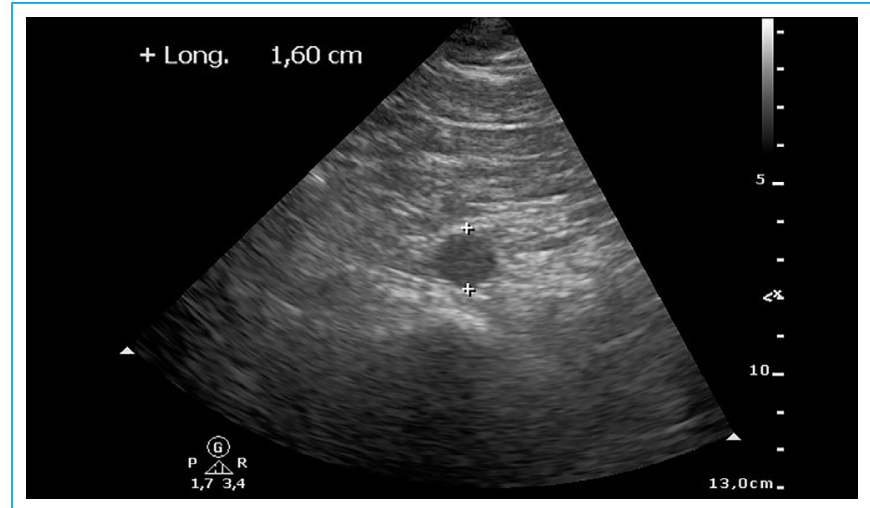

Figura 4. Corte transverso de Aorta Abdominal con medición de borde externo a externo (antero-posterior). Note el uso de transductor cardiaco

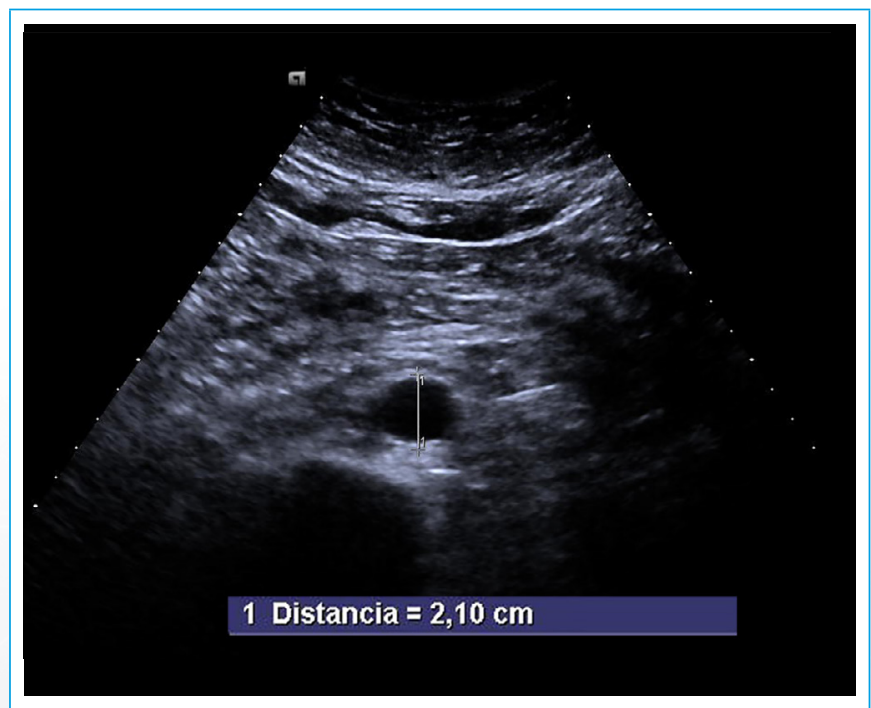

Figura 5. Medición de diámetro antero-posterior incluyendo las paredes aórticas

\section{Ideas para recordar}

1. El eco Doppler permite valorar de forma rápida y sencilla la aorta abdominal.

2. La aorta abdominal se mide de borde externo a borde externo. El diámetro máximo normal es $20 \mathrm{~mm}$ en adulto.

\section{Bibliografía}

1. Ricci M, Kleeman M, Case T, Pilcher D: Normal aortic diameter by ultrasound. J Vasc Technol 1995; 19:17-19.

2. Zwiebel W. Ultrasonografía Vascular $4^{\circ}$ edición, 2002.

3. Lederle F, Johnson G, Wilson S, et al. Relationship of age, gender, race and body size to infrarenal aortic diameter.J Vasc Surg 1997; 25:595-601.

4. Verloes A, Sakalihasan N, Koulischer L, Limet R. Aneurysms of the abdominal aorta: familial and genetic aspects in three hundred thirteen pedigrees. J Vasc Surg 1995; 21:646-51.

5. Perea G, Corneli M, Elisamburu P, Casas J, et al. Consenso de Ecografía Doppler Vascular. Rev Argent Cardiol 2020; 88 (Suplemento 4).

6. Perea G, Corneli M. E-Non-invasive techniques in diagnosis and treatment of patients with abdominal aortic aneurysm. Journal-of-Cardiology-Practice 2020; Vol. $18, N^{\circ} 32-26$ Aug 2020

7. Corneli M; Perea G. Which patients should we screen to detect an aneurysm of the abdominal aorta?. E-Journal-of-Cardiology-Practice/ Vol. $18, N^{\circ} 29-29$ Jul 2020. 